\title{
Propositions d'aménagement du jaugeage chimique des débits des rivières Méthode utilisant les colorants alimentaires
}

\author{
PAR
}

\author{
Ph. Lion \\ ET \\ G. Galea \\ Ingénieur chimiste \\ Ingénieur hydrogéologrue \\ a la Division Hydrologie du C..'A.G.R.E.F. (*)
}

\section{Remarques préliminaires}

Les méthodes chimiques de détermination du débit des rivières ont pris au cours des demières décennies un remarquable essor en raison de leur simplicité, de leur rapidité, de leur précision et de leur prix modéré lorsque les débits ne sont pas trop élevés. Utilisant la méthode par injection à débit constant sur le bassin représentatif de l'Orgeval, nous avons été amenés à faire certaines remarques qu'il a semblé intéressant de publier, afin d'apporter notre contribution au perfectionnement du procédé.

1.1. Le marqueur le plus couramment utilisé est le dichromate de sodium. Quels que soient les avantages que présente ce corps : produit courant, grande solubilité, facilité de dosage, prix modéré, etc.... son choix est discutable. Il présente en effet l'inconvénient de faire partie des produits toxiques dont l'emploi est règlementé. L'AFNOR a d'ailleurs tenu compte de cette règlementation en annulant, à la date du 15 novembre 1975 , la norme expérimentale X10-305 "Mesure de débit de l'eau dans les cheneaux - Méthodes de dilution utilisant des traceurs non radioactifs" qui recommandait entre autres l'emploi du dichromate de sodium.

Il apparait donc hautement souhaitable de substituer au dichromate de sodium un traceur exempt de toxicité.

1.2. Divers procédés ont été préconisés - et utilisés pour permettre les mesures de débits en eaux chargées (filtration, centrifugation) et pour réduire la quantité

(*) Centre Technique du Génie Rural des Eaux et des Forêts Parc de Tourvoie - 92160 - ANTONY de colorant à mettre en cuvre (extraction), en vue d'abaisser le coût du jaugeage des grands débits.

a) La filtration sur papier doit être prohibée en raison du risque de rétention de colorant signalé par Marichal et Benoit (1962), auquel peuvent s'ajouter d'autres causes d'erreur, comme par exemple la réduction partielle en leucodérivé, qui peut entraîner un affaiblissement plus ou moins prononcé de la couleur, voire un changement de teinte. Par contre, on peut envisager une filtration sur entonnoir à plaque filtrante en verre fritté, qui peut être effectuée rapidement et simplement sous vide (une trompe à eau suffit), sans altérer la solution colorée.

b) La centrifugation proposée par Marichal et Benoit (1962) ne doit pas être retenue. D'une part, ces auteurs envisagent d'opérer sur des échantillons de 21 d'eau, ce qui nécessite une centrifugeuse de grandes dimensions comme ne peut en posséder qu'un important laboratoire. D'autre part, cette opération, multipliée par le nombre généralement assez grand d'échantillons, constituerait une lourde charge pour le manipulateur. Enfin, comme il vient d'être indiqué en a), la filtration sur verre fritté permet đ'obtenir sans difficulté le résultat cherché.

c) Plusieurs auteurs - même parmi ceux dont les travaux ont le plus contribué à promouvoir les méthodes de jaugeage chimique - ont proposé des procédés de concentration des solutions diluées, soit par extraction du colorant, soit plus rarement par évaporation de l'eau. C'est ainsi qu'André (1969) mentionne des "nécessités de reconcentration" et précise que l'on peut procéder à une extraction par le butanol dans le cas du dichromate de sodium. Il signale, d'ailleurs, l'imprécision à laquelle conduit ce procédé. De son côté, Dumas (1953), parle d'évaporation. Marichal et Benoit (1962) utilisent, au cours du dosage de la rhodamine B conseillée, entre autres, 
pour les mesures de débits, une extraction par l'isopentanol. L'extraction est également indiquée par Burelli et Ecrement (1969) dans le cas de jaugeages de grands débits.

L'extraction est à déconseiller pour plusieurs raisons. Tout d'abord, ce n'est pas une méthode "quantitative", c'est-à-dire qu'elle ne permet pas d'appréhender la totalité du colorant présent dans la solution, contrairement à ce qu'admettent implicitement les auteurs précités, quand ils ne vont pas jusqu'à affirmer, comme Marichal et Benoit (1962), qu'une extraction unique suffit pour retirer la totalité du colorant de sa solution aqueuse. Certes, le coefficient de partage du colorant entre l'eau et le solvant extracteur est largement en faveur de ce dernier, et l'on peut admettre qu'au bout de trois extractions la quantité qui reste dans l'eau est négligeable, mais alors on complique singulièrement le jaugeage chimique et l'on augmente notablement sa durée. D'autre part, il est matériellement impossible d'opérer de façon identique pour tous les échantillons, de sorte que l'extraction constitue une source d'imprécision.

1.3. La méthode d'analyse comporte généralement l'élavoration plus ou moins compliquée d'un complexe. Dans le cas du dichromate de sodium, il faut faire agir la diphénylcarbazide en solution acétonique, dans des conditions strictes de $\mathrm{pH}$. Comme l'indique par ailleurs Dodero (1953), la coloration se développe lentement et l'on ne peut jamais être sûr que la mesure est effectué au bon moment. La formation du complexe est une opération relativement longue et délicate, et là encore on peut craindre qu'il ne s'introduise entre les divers échantillons, et quel que soit le soin apporté, des différences de traitement qui se répercuteront sur les résultats.

Cette dernière remarque ne s'applique pas à la fluorescéine dont la couleur est déterminée directement sur les solutions, mais ce colorant a malheureusement le double inconvénient d'être assez fortement adsorbé par les matières en suspension, les berges et le fond, et d'être instable à la lumière.

1.4. Le marqueur doit - entre autres qualités - être facilement soluble dans l'eau, et ses solutions doivent se mélanger rapidement à l'eau de la rivière. Si un colorant, intéressant par ailleurs, se dissout mal ou que ses solutions se mélangent mal aux eaux naturelles; on peut lever cette difficulté par l'incorporation à la solution-mère d'un surfactif judicieusement choisi (Hlavek 1973). On évitera l'emploi de cationiques en raison de leur toxicité, et l'on s'adressera suivant le cas aux anioniques (alcools gras à chaîne droite sulfatée sodique, par exemple), ou aux non ioniques (alkylphénols polyoxyéthylénés ou autres).

1.5. Dans le même ordre d'idées - bonne dispersion du marqueur - on pourrait poursuivre les essais de dispersion partielle à l'injection, malgré les médiocres résultats obtenus jusqu'ici avec les systèmes de pommes d'arrosoir ou de tube horizontal perforé. Certains auteurs (Dumas 1953) ont depuis longtemps insisté pour une injection répartie sur plusieurs points quand la turbulence est trop faible pour que l'injection ponctuelle suffise à assurer un mélange rapide.
1.6. Etant donné l'importance que présente pour le calcul final la connaissance précise du débit d'injection, il est essentiel que les diaphragmes soient minutieusement calibrés. Cette question a déjà été traitée (Bailleux 1974) et n'est évoquée que pour mémoire.

1.7. La technique utilisée en laboratoire doit être soigneusement mise au point. Comme elle devra presque toujours être pratiquée par des hydrologues ou des techniciens qui n'auront qu'une habitude limitée des appareils de chimie et de physico-chimie, elle ne devra faire appel à aucune des méthodes, à aucun des instruments qui recèlent des pièges dans lesquels pourraient tomber même des chimistes ou des physiciens de formation.

Il y aura lieu, entre autres, au laboratoire :

a) de préférer, pour la courbe d'étalonnage, le système des dilutions successives utilisé à l'origine, par exemple par Dodero (1953), à l'emploi d'une micropipette qui peut conduire à des erreurs graves aux très faibles débits, voire au décalage de la droite d'étalonnage ; il faut bien entendu veiller alors à l'exactitude des récipients jaugés utilisés et se méfier des pipettes à système d'aspiration non retarées;

b) de déterminer avec le plus grand soin deux points, encadrant d'aussi près que possible la valeur moyenne des lectures obtenues avec les échantillons, afin de réduire au minimum l'erreur commise en interpolant entre ces deux points

c) d'utiliser un spectrophotomètre aussi sensible que possible ; pour améliorer encore cette sensibilité, on prendra pour déterminer le zéro non de l'eau de la rivière, mais une solution de coloration plus faible que les échantillons prélevés, et on règlera le 100 de l'échelle sur une solution de coloration convenable. Ces deux solutions auront été préparées en diluant de façon appropriée une prise de la solution injectée avec de l'eau prélevée dans la rivière.

Moyennant ces précautions, on limitera l'imprécision, et l'on pourra abaisser notablement la concentration finale du colorant, donc sa consommation.

Dans les opérations effectuées sur le terrain, il semble possible d'apporter une certaine amélioration à la méthode chimique de mesure des débits des cours d'eau :

1.8. en utilisant des diaphragmes parfaitement calibrés, afin d'obtenir un débit d'injection du marqueur qui soit régulier et connu avec précision ;

1.9. en incorporant un surfactif à la solution injectée, afin que la dispersion du marqueur soit rapide et complète, et que l'influence des eaux mortes soit diminuée ;

1.10. en choisissant un marqueur exempt de toxicité, tant pour l'homme que pour la faune et la flore.

La question des diaphragmes a déjà été étudiée (Bailleux 1974). L'emploi d'un surfactif (Hlavek 1973) constitue une amélioration certaine, mais le choix ne peut en être fait avant celui du marqueur, ce dernier risquant d'être incompatible avec un surfactif prédéterminé.

Chronologiquement, le point 3 doit donc être réglé avant le point 2. C'est le principal objet de la présente étude. 
Laissant de côté l'utilisation des isotopes qui met en oeuvre un appareillage particulier, exige un personnel spécialisé et, dans le cas des isotopes radioactifs, nécessite des formalités plus ou moins compliquées, il a semblé intéressant de reprendre entièrement la question dans ses modalités, et de l'étudier de façon systématique. On a donc examiné successivement les possibilités d'emploi :

- des différents éléments minéraux ;

- des sels minéraux antérieurement proposés et mis en ouvre ;

- des colorants organiques déjà utilisés ;

- des colorants alimentaires qui, à notre connaissance, n'ont jamais été proposés bien qu'ils semblent tout indiqués.

L'étude théorique a été suivie d'essais pratiques dont les résultats sont communiqués, l'ensemble conduisant à la proposition concrète de substituer de nouveaux marqueurs au dichromate de sodium.

\section{Etude théorique}

\subsection{Eléments minéraux}

\subsubsection{Idée directrice de la recherche}

Le remplacement du dichromate de sodium couramment utilisé étant commandé par la toxicité du chrome hexavalent, comme il a été indiqué au début de cette étude (I - Remarques préliminaires, 1.1), le premier critère dans la recherche du marqueur est naturellement son innocuité. Les autres critères sont les mêmes que ceux qui ont conduit au choix des divers agents utilisés jusqu'à présent :

- absence du milieu naturel ;

- facilité de dosage ;

- prix de revient acceptable

Afin d'effectuer une recherche aussi rigoureuse que le permettent les renseignements assez incomplets dont on peut disposer sur la fréquence des éléments dans les eaux naturelles et leurs effets sur la faune aquatique et sur l'homme, il a semblé commode de suivre le tableau de Mendeleieff et d'éliminer systématiquement :

- les éléments toxiques ou radioactifs ;

- les éléments couramment présents dans les eaux naturelles ;

- les éléments rares ou de prix élevé ;

- les éléments dont le dosage est particulièrement compliqué ou mal connu.

\subsubsection{Etude du tableau de Mendeleieff}

Les critères précédents ont conduit à éliminer presque tous les éléments du tableau. On aurait pu à la rigueur retenir l'aluminium, l'iode et le cobalt. Toutefois, même ces trois éléments ne donnent pas entière satisfaction.

L'aluminium, commercialisé sous des formes multiples, est relativement bon marché. Il est réputé sans danger pour la faune aquatique et son dosage ne présente pas de difficultés. Il pourrait donc être mis en ouvre pour le jaugeage chimique des débits de rivières. Toutefois, la facilité avec laquelle il précipite exclut toute garantie de précision dans la détermination du rapport des concentrations initiale et finale.

L'iode peut présenter des risques d'adsorption ou de complexation, en particulier dans les zones argileuses ou en cas de rejets de sels de métaux trivalents.

Quant au cobalt, il risque - même à très faible concentration - d'avoir soit directement, soit en tant que constituant de la vitamine $\mathrm{B} 12$, un effet catalytique sur certaines réactions favorables à l'eutrophisation. Il semble donc préférable de ne pas l'introduire dans le milieu naturel.

On est alors logiquement conduit :

- soit à essayer d'améliorer l'une des méthodes déjà utilisées ;

- soit à rechercher un procédé différent

\subsection{Retour sur les méthodes classiques}

Si l'on se reporte aux travaux antérieurs, on constate que les sels effectivement utilisés sont assez peu nombreux. L'une des principales études (Dumas, 1953) indique à côté du dichromate de sodium : le chlorure de sodium et le sulfate de manganèse. Ce dernier sel mérite de retenir l'attention. Si le manganèse n'est pas toujours absent des eaux naturelles, puisque Berzelius trouva jusqu'à $7 \mathrm{mg} / \mathrm{l}$ de carbonate de manganèse dans les eaux de Carlsbad et qu'une source voisine de Kansas en contiendrait environ $10 \mathrm{mg} / \mathrm{l}$ (Pascal 1934), c'est néanmoins un élément qu'on y rencontre rarement. Le sulfate de manganèse est un produit courant, qui se dissout bien dans l'eau, et le manganèse peut être dosé sans difficulté avec une bonne précision.

Certes, le manganèse - sans être à proprement parler un élément toxique - est classé comme "indésirable" par la règlementation française (arrêté du 10 Août 1961), qui recommande pour les eaux potables une teneur inférieure à $0,1 \mathrm{mg} / \mathrm{l}$. Mais cette valeur n'exclut nullement l'emploi du sulfate de manganèse pour le jaugeage chimique. Un mode opératoire très complet, a été décrit et commenté entre autres par Pruvot(1953) qui l'a proposé à cette fin dès 1925 comme l'indique Bouvard (1950).

On pourrait également envisager l'emploi du cuivre, élément "indésirable" comme le manganèse, mais dont le seuil est dix fois plus élevé : $1 \mathrm{mg} / 1$ dans l'eau potable, et qui comme le manganèse est assez facile à doser par spectrophotométrie (Norme NF'T 90 - 022).

Pruvot (1953) dosait le sulfate de manganèse par voie chimique. Il conseillait d'injecter une solution à environ $100 \mathrm{~g} / 1$ et de ne pas descendre, pour la solution diluée, au-dessous de $2,5 \mathrm{mg} / \mathrm{l}$. Si cette limite correspondait en effet à ce qu'on pouvait attendre des méthodes utilisées à l'époque, le dosage spectrophotométrique permet aujourd'hui de déterminer une concentration cent fois plus basse (Norme NF T 90-024). La masse de sel à mettre en oeuvre peut évidemment être réduite dans le même rapport, ce qui rend le procédé particulièrement rentable. Si l'on prend un sel de cuivre, on peut également en utiliser des quantités assez faibles, le dosage spectrophotométrique permettant de déterminer des concentrations inférieures à $50 \mu \mathrm{g} / 1$ - moyennant, il est vrai, un traitement un peu compliqué - (Norme NF T 90-022 Additif). 


\subsection{Colorants déjà utilisés}

Des essais ont été effectués en France avec la rhodamine B (Rodier, Marichal 1961). Toutefois, l'emploi de ce colorant rouge peu toxique est lié à certains inconvénients. D'une part, il est assez peu soluble dans l'eau : environ $10 \mathrm{~g} / 1$ à température ordinaire. On a donc cherché divers solvants permettant d'injecter des solutions plus concentrées. Cormary (1968) indique entre autres : le méthanol pur, qui dissout jusqu'à $800 \mathrm{~g} / 1$ de rhodamine $B$; le mélange éthanol-eau 40/60, qui dissout environ $200 \mathrm{~g} / 1$; l'héthanol : $90 \mathrm{~g} / \mathrm{l}$. Pour sa part, Cormary a utilisé le mélange eau éthanoïque $50 / 50$ qui permet de dissoudre $80 \mathrm{~g} / \mathrm{l}$ de rhodamine $\mathrm{B}$, quantité suffisante pour ses essais. La rhodamine $B$ présente un autre inconvénient pour l'emploi dans les eaux naturelles: elle est plus ou moins fortement adsorbée par les matières en suspension, les fonds et les rives comme l'a montré, entre autres, Lallemand (1964). Le dosage de la rhodamine B exige par ailleurs l'emploi d'un fluorimètre. Certains auteurs (Marichal, Benoit 1962) ont même proposé des méthodes de dosage assez compliquées pour augmenter la sensibilité des mesures, en vue de réduire les quantités à mettre en ouvre pour le jaugeage des débits. La complexité des méthodes proposées exige alors la disposition d'un laboratoire bien équipé et de personnel spécialisé et ces méthodes devraient d'ailleurs être modifiées pour donner des résultats précis. Aux U.S.A., on utilise la rhodamine $W_{t}$ pour le contrôle continu des débits, comme l'indique André (1969). Ce colorant semble d'un emploi plus commode que la rhodamine $B$. Toutefois, les américains indiquent qu'il ne donne en jaugeage continu qu'une précision de 5 à $10 \%$, que l'autonomie du procédé n'est que d'une semaine et exige une cuve de 1001 , et qu'enfin l'injection continue de très faibles quantités de solution pose des problèmes délicats (André 1969).

Des études en cours, effectuées par le C.E.N.G. à l'instigation du Service de l'Hydraulique du Ministère de l'Agriculture, permettront peut-être de les résoudre.

\subsection{Marqueurs proposés}

La législation française autorise l'incorporation aux aliments ou aux boissons d'un certain nombre de colorants dits "alimentaires". Ces produits sont considérés comme exempts de toxicité pour l'homme, et on peut penser jusqu'à preuve du contraire qu'ils sont également sans danger pour la faune aquatique et les végétaux. Il suffirait donc de trouver parmi ces colorants alimentaires un produit :

- très soluble dans l'eau, ce qui est le cas de tous les colorants pour les boissons non alcoolisées;

- stable dans l'intervalle de pH 6,5-8,5, ce qui est toujours le cas ;

- stable à la lumière, ce qui est l'une des caractéristiques des colorants alimentaires de bonne qualité ;

- non adsorbable par les matières en suspension, le fond et les rives ;

- susceptible d'un dosage fin à de très faibles concentrations.

Cette solution aurait en outre l'avantage que le colorant pourrait servir aux essais préliminaires d'évaluation de la durée du trajet du point d'injection au point de prélèvement, et de la durée de passage en ce dernier point. Il serait toutefois prudent, pour éviter les risques de retenue du colorant par les eaux mortes et de lâcher intempestif, de choisir, pour les essais préliminaires et le dosage, deux colorants de couleurs nettement différentes (donc de longueurs d'onde optimales éloignées).

Les considérations précédentes nous ont conduits à sélectionner, dans la très vaste gamme des colorants alimentaires, les trois produits suivants $\left({ }^{1}\right)$ :

$\begin{array}{ll}\text { TARTRAZINE } & \text { E } 102 \\ \text { JAUNE SOLEIL } & \text { E } 110 \\ \text { COCCINE NOUVELLE } & \text { E } 124\end{array}$

Outre leur parfaite innocuité, ces colorants présentent les avantages suivants :

- assez grande solubilité ;

- pouvoir colorant intense ;

- dosage direct, c'est-à-dire ne nécessitant pas la formation d'un complexe.

Le fournisseur des produits étudiés, qui dose couramment par spectrophotométrie des solutions très diluées de ces colorants, a bien voulu nous communiquer pour chacun des trois corps retenus les courbes qui sont reproduites en annexe :

- courbe de densités optiques en fonction de la longueur d'onde, qui fait apparaître les longueurs optimales suivantes (Fig. 1,3 et 5) :

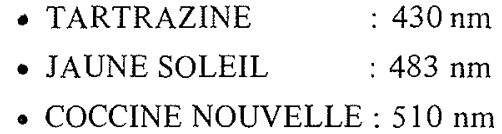

- courbe de densités optiques en fonction de la concentration des solutions, qui montre (Fig. 2, 4 et 6):

a) que la loi de Beer-Lambert s'applique remarquablement aux dilutions étudiées $(\mathrm{C}<25 \mathrm{mg} / \mathrm{l})$;

b) que, toutes choses égales d'ailleurs, on obtiendra probablement de meilleurs résultats avec le Jaune Soleil qu'avec la Tartrazine et avec la Tartrazine qu'avec la Coccine, les densités optiques correspondant, par exemple, à des concentrations de $10 \mathrm{mg} / \mathrm{l}$ étant respectivement :

- pour la TARTRAZINE : :0,40

- pour le JAUNE SOLEIL : : 0,92

- pour la COCCINE NOUVELLE : 0,29

Du point de vue de la concentration d'emploi, ces colorants sont comparables au dichromate de sodium. Les jaugeages chimiques effectués avec le dichromate ont montré qu'on obtient de bons résultats lorsque la concentration en sel des échantillons prélevés est de l'ordre de $0,5 \mathrm{mg} / \mathrm{l}$. Il en est de même avec les trois colorants alimentaires ci-dessus. Nous avons même eu d'excellents résultats avec des concentrations finales nettement plus faibles, de l'ordre du dixième de milligramme par litre.

Quant au prix de revient, il est plus élevé. Les prix hors taxes au kilogramme sont de l'ordre de $5 \mathrm{~F}$ pour

(1) Les produit's utilisés provenaient de la Sté Metayer Aromatique S.A. $7 \& 9$, rue Gambetta à Champigny (94500) 


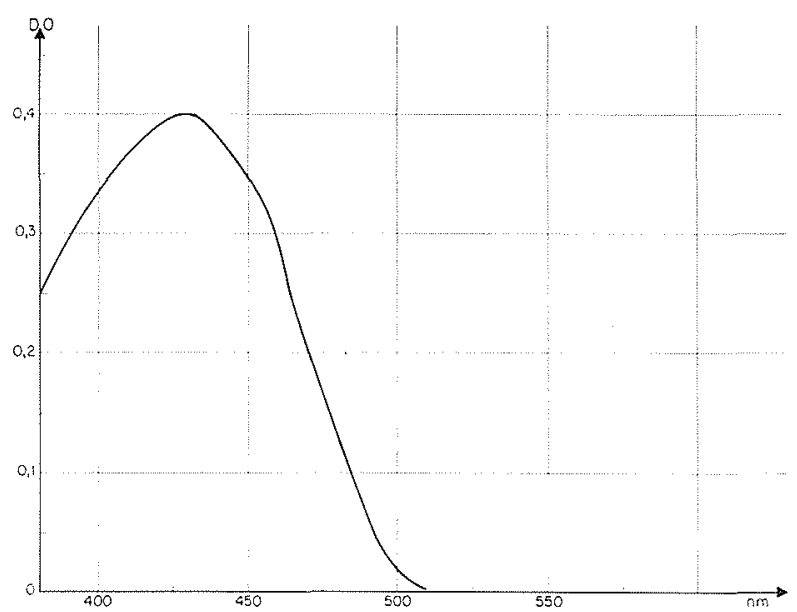

1/ Tartrazine sol. 10 mg/l. (d'après Métayer-Aromatique S.A.)

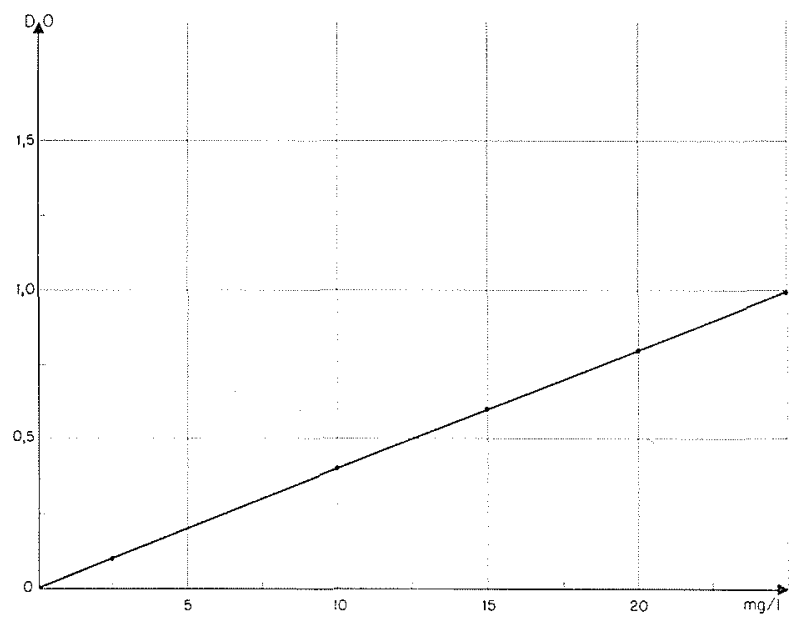

2/ Tartrazine $\lambda=430 \mathrm{~nm}$ ( $d$ 'après Métayer-Aromatique S.A.)

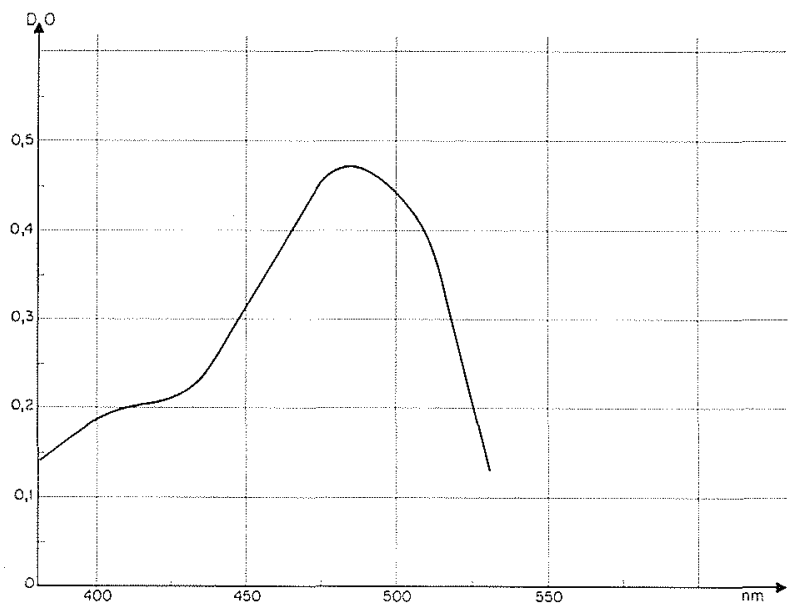

3/Jaune-Soleil sol. $10 \mathrm{mg} / \mathrm{l}$ (d'après Métayer-Aromatique S.A.)

le dichromate de sodium technique, et de $60 \mathrm{~F}$ pour les colorants alimentaires étudiés $\left({ }^{2}\right)$. En opérant comme il vient d'être dit, de façon à avoir une concentration finale de $0,1 \mathrm{mg} / 1 \mathrm{de}$ colorant alimentaire, on a donc très sen-

(2) Ce prix est réduit à $40 \mathrm{~F}$ pour une commande de $1 \mathrm{t}$.

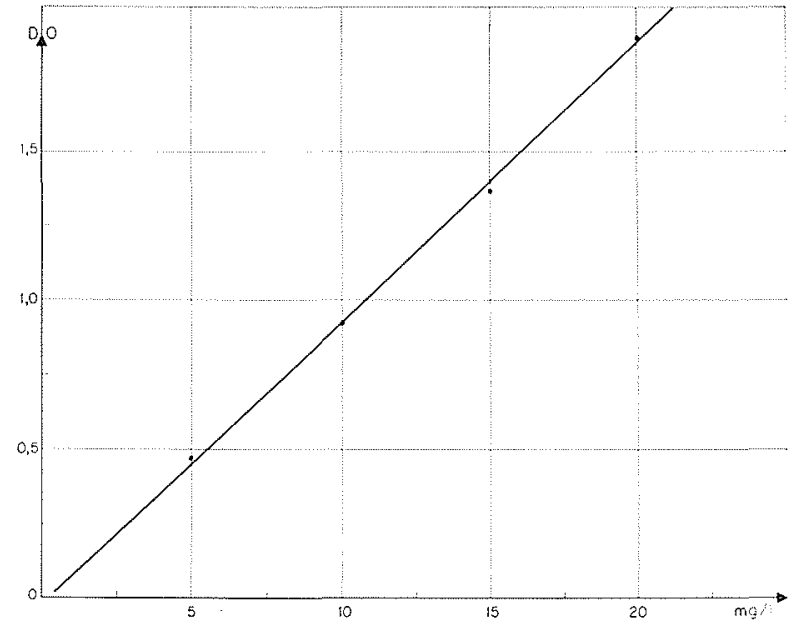

4/ Jaune-Soleil $\lambda=483 \mathrm{~nm}$ (d'après Métayer-Aromatique S.A.)

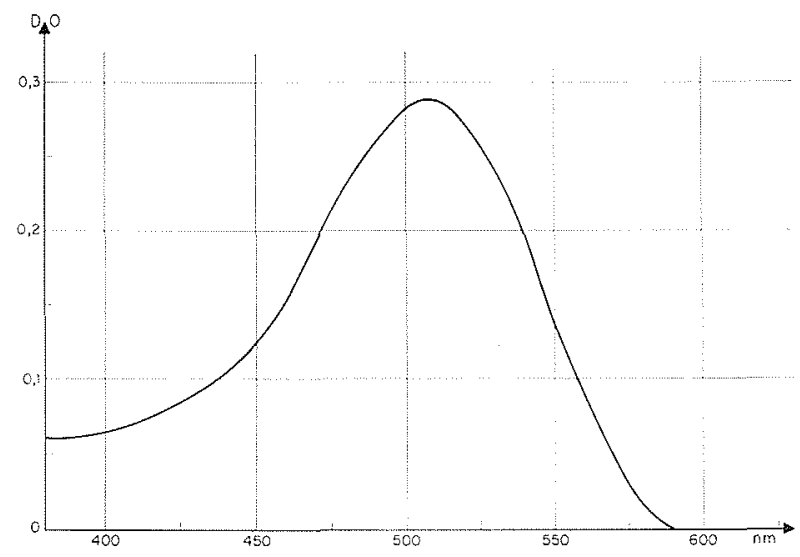

5/ Coccine Nouvelle sol. $10 \mathrm{mg} / \mathrm{l}$ (d'après Métayer-Aromatique S.A.)

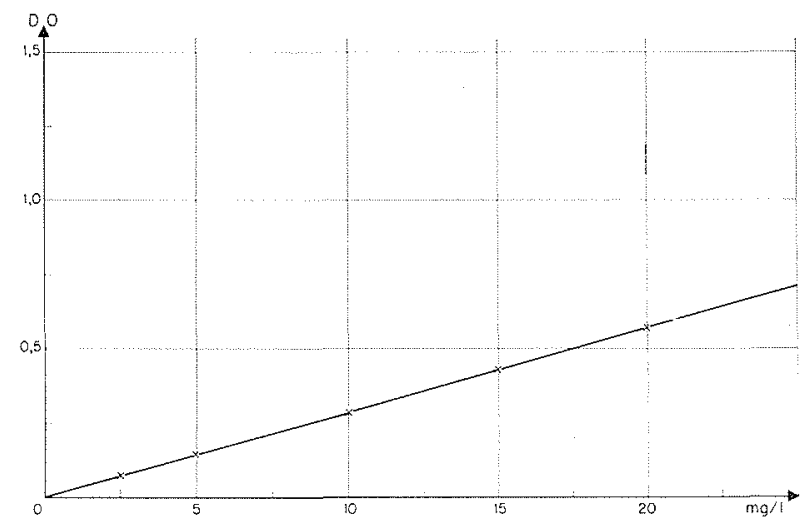

6/ Coccine Nouvelle $\lambda=510 \mathrm{~nm}$ (d'après Métayer-Aromatique S.A.)

siblement une dépense double, étant entendu que la concentration finale du dichromate est de l'ordre de $0,5 \mathrm{mg} / \mathrm{l}$. Il ne faut pas oublier cependant l'économie réalisée en n'utilisant aucun autre produit tel que la diphénylcarbazide, l'acétone, l'acide sulfurique et le solvant extracteur éventuel. 
Il est possible d'utiliser des colorants qui permettent d'effectuer des dosages plus fins (des colorants bleus ou verts, par exemple), mais leur prix étant plus élevé, cela ne constitue aucun avantage économique. D'autre part, il serait maladroit, même si cela permettait d'abaisser très largement le coût du jaugeage, de faire appel à un colorant d'importation, plus ou moins difficile à se procurer, et dont le prix risque de fluctuer de façon imprévisible et peut-être importante avec le change. Il est préférable d'affiner la méthode de dosage - sans la compliquer pour autant - comme il est indiqué dans la troisième partie.

\section{Etude pratique}

Les trois colorants alimentaires précités ayant été choisis en raison des caractéristiques indiquées par le fabricant, il était nécessaire de procéder à des essais sur le terrain. Il fallait pour cela disposer de cours d'eau dont le débit soit parfaitement connu ou puisse être déterminé avec la précision maximale par d'autres méthodes. Aussi des essais pratiques ont-ils été effectués sur les bassins représentatifs de l'Orgeval qui répondent à ces conditions et ont été largement étudiés et décrits par exemple par Hlavek (1967), Hlavek, Cambon, et Zumstein (1968).

\subsection{Première série d'essais}

L'équipe de jaugeage comprenait, outre les deux signataires, le chef de la Station de Boissy, excellent technicien parfaitement rompu aux opérations de jaugeage chimique, et son adjoint.

Nous avons procédé au jaugeage chimique par injection continue à débit constant et à titre de contrôle un jaugeage au moulinet a été réalisé simultanément dans une section canalisée et donc parfaitement connue.

Voici à titre d'exemple deux des résultats obtenus

\begin{tabular}{|c|c|c|c|c|c|}
\hline Lieu & Date & Colorant & $\begin{array}{l}\text { Jaugeage } \\
\text { chimique }\end{array}$ & Moulinet & Ecart $^{(*)}$ \\
\hline La Gouge & 18.06 .74 & $\begin{array}{l}\text { Jaune Soleil } \\
\text { Sol. à } 1 \mathrm{~g} / 1\end{array}$ & $36,25 \mathrm{l} / \mathrm{s}$ & $35,15 \mathrm{l} / \mathrm{s}$ & $3 \%(1)$ \\
\hline Le Theil & 11.07 .74 & $\begin{array}{l}\text { Tartrazine } \\
\text { Sol. à } 0,5 \mathrm{~g} / \mathrm{l}\end{array}$ & $85,5 \quad 1 / \mathrm{s}$ & $83,9 \quad 1 / \mathrm{s}$ & $2 \%(2)$ \\
\hline
\end{tabular}

(*) Il ne s'agit pas d'un calcul d'erreus par rapport au débit réel de la rivière mais de l'écart relatif d'un procédé par rapport à l'autre.

(1) Il importe de remarquer que ce jaugeage chimique a été effectué dans les conditions les plus défavorables, un débit aussi faible dans un lit large de 3 à 4 mètres ne remplissant certainement pas les conditions optimales d'application de la méthode.

(2) Dans une étude concemant l'emploi de la rhodamine $B$ Cormary (1968) indique $2 \%$ comme écart maximal entre les résultats du jaugeage au moulinet et du jaugeage chimique par injection continue. On peut donc considérer comme valable ce jaugeage aux colorants alimentaires, d'autant plus que le débit mesuré était très faible.

\subsection{Deuxième série d'essais}

L'emploi des colorants alimentaires donnant de bons résultats pour des débits inférieurs à $1001 . \mathrm{s}^{-1}$, il était nécessaire de passer à des débits plus importants. Dans une deuxième série d'essais, on testa la méthode pour des débits de l'ordre de $2 \mathrm{~m}^{3} \cdot \mathrm{s}^{-1}$. Les essais ont eu lieu à Aix les Bains, sur le Sierroz, avec l'aide du personnel du Laboratoire du Petit Port (Division Qualité des Eaux, Pêche et Pisciculture du CTGREF). Les prélèvements étaient effectués d'un poncelet situé à l'amont immédiat d'une échelle limnimétrique. L'opération eut lieu le 11 février 1975. On effectua dans la matinée un jaugeage à la tartrazine, et l'après-midi un jaugeage au dichromate, méthode très bien connue du personnel. Les résultats furent les suivants :

\begin{tabular}{|c|c|c|c|c|c|}
\hline Lieu & Date & Colorant & Jaugeage & Contrōle & Ecart \\
\hline Aix les Bains & 11.02 .75 & $\begin{array}{l}\text { Tartrazine } \\
12,5 \mathrm{~g} / 1\end{array}$ & $1750 \mathrm{l} / \mathrm{s}$ & $1800 \mathrm{l} / \mathrm{s}$ & $<3 \%$ \\
\hline
\end{tabular}

Dans cette opération, la solution injectée était à environ $12,5 \mathrm{~g} \cdot 1^{-1}$ et les échantillons prélevés à un peu plus de 0,1 mg. $1^{-1}$. Il est possible de descendre à des concentrations plus faibles. Avec un bon spectrophotomètre, on peut certainement avoir une précision satisfaisante avec des concentrations deux fois plus faibles et jauger des débits de l'ordre de $10 \mathrm{~m}^{3}$ en n'utilisant pas plus de $5 \mathrm{~kg}$ de tartrazine.

\subsection{Troisième série d'essais}

Pour la troisième série d'essais, le Service Régional d'Aménagement des Eaux Languedoc-Roussillon a bien voulu nous accueillir et mettre à notre disposition son équipe de jaugeage et son matériel. Le débit, au jour de la mesure, était un peu inférieur aux $10 \mathrm{~m}^{3} / \mathrm{s}$ escomptés. Dans ce cas aussi, les résultats furent satisfaisants :

\begin{tabular}{|c|c|c|c|c|c|}
\hline Lieu & Date & Colorant & Jaugeage & Contrôle & Ecart \\
\hline Ganges & 29.04 .75 & Tartrazine & $6,8 \mathrm{~m}^{3} / \mathrm{s}^{(1)}$ & $7,2 \mathrm{~m}^{3} / \mathrm{s}^{(2)}$ & $<6 \%$ \\
\hline
\end{tabular}

(1) Jaugeage effectué le matin (colorant : tartrazine)

(2) Jaugeage effectué l'après-midi (colorant : dichromate de sodium).

(3) Entre le matin et l'après-midi le niveau à l'échelle limnimétrique était monté de quelques millimètres.

L'écart des débits trouvés par les deux méthodes est de l'ordre de $6 \%$. Toutefois, compte tenu de l'augmentation de débit survenue entre les deux mesures, on peut considérer ces résultats comme une nouvelle confirmation de la validité de la méthode à la tartrazine. D'autre part, on ne disposait que d'un spectrophotomètre Jean et Constantqui, compte tenu des dilutions obtenues, ne permettait pas des lectures dans un domaine de fonctionnement suffisamment fiable.

\section{Résultat de l'étude}

La toxicité đu chrome hexavalent incitait à rejeter l'emploi du dichromate de sodium pour les jaugeages chimiques, sans attendre que la réglementation l'interdise. La recherche systématique n'a pas permis de trouver un autre sel qui donne entièrement satisfaction. Les colorants alimentaires étant exempts de toxicité, il était logique de proposer leur emploi, mais nécessaire de vérifier auparavant 
s'ils étaient utilisables des points de vue technique et économique. L'étude que nous venons de résumer $\left({ }^{3}\right)$ a permis de constater que les trois colorants alimentaires proposés sont opérationnels pour les débits faibles et moyens.

La concentration de la solution-mère peut être choisie, suivant le débit à mesurer, entre 0,5 et $50 \mathrm{~g} / 1$, soit un domaine de concentration allant de 1 à 100 . La solubilité des trois colorants étudiés est de l'ordre de 80 à $100 \mathrm{~g} / 1$. Si l'on veut jauger des débits importants, on peut -à défaut d'une solution- utiliser une suspension plus riche. Des essais ont montré qu'il est possible à l'aide de divers artifices (modification du $\mathrm{pH}$, addition d'un surfactif ou autre) d'obtenir une suspension homogène et stable jusqu'à $250 \mathrm{~g} / 1$.

Sur le terrain, le mode opératoire décrit dans la littérature peut être utilisé sans modification, exception faite de la possibilité déjà signalée de substituer un colorant alimentaire à la fluorescéine pour déterminer le temps d'arrivée au point de prélèvement et le temps de passage.

$\mathrm{Au}$ laboratoire, les opérations sont considérablement simplifiées parce qu'il n'y a pas besoin de former un complexe. Le processus opérationnel peut être schématisé comme suit.

\section{a) Optimisation de l'échelle}

Préparer à partir d'un échantillon de la solution injectée des dilutions de concentrations rondes (multiples de dix millièmes, de cent millièmes, de millionièmes, etc. . . suivant le cas) qui encadrent les échantillons recueillis aux points de prélèvements. Régler respectivement le zéro et le cent de la graduation du spectrophotomètre sur deux dilutions encadrant les échantillons, en se conformant aux instructions du constructeur.

\section{b) Courbe d'étalonnage}

Par dilutions successives, préparer des solutions dont la concentration est dans un rapport simple avec celle de la solution injectée. Par exemple : $10^{-6}, 2.10^{-6}, 3.10^{-6}$. Reporter sur un papier millimétré les lectures correspondant à chacun de ces rapports.

\section{c) Echantillons}

Lire les valeurs de l'échelle correspondant aux échantillons. Eliminer les valeurs aberrantes éventuelles, faire la moyenne des valeurs retenues, soit $\mathrm{m}$.

\section{d) Détermination du débit $Q$}

Reporter m en ordonnée sur la courbe d'étalonnage b). Il lui correspond, sur le segment de droite $\left({ }^{4}\right)$ qui joint les deux points de la courbe d'étalonnage qui l'encadrent, un point dont l'abscisse représente la concentration relative $\mathrm{r}=\mathrm{c} / \mathrm{C}$. Connaissant le débit $\mathrm{q}$ du diaphragme utilisé, on en déduit $Q$ par la relation $Q=q / r$ (fig. 7).

(3) L'étude détaillée a fait l'objet d'une autre publication : Le jaugeage chimique des débits des rivières - Propositions d'aménagements de la méthode classique, par $\mathrm{Ph}$. Lion et $\mathrm{G}$. Galea. Mémoire $n^{\circ} 3$ - Ministère de l'Agriculture - C.T.G.R.E.F. - Décembre 1975.

(4) A-défaut, sur la droite ajustée à tous les points d'étalonnage si ceux-ci sont trop dispersés pout qu'on puisse faire confiance aux seuls 2 points qui encadrent $m$.

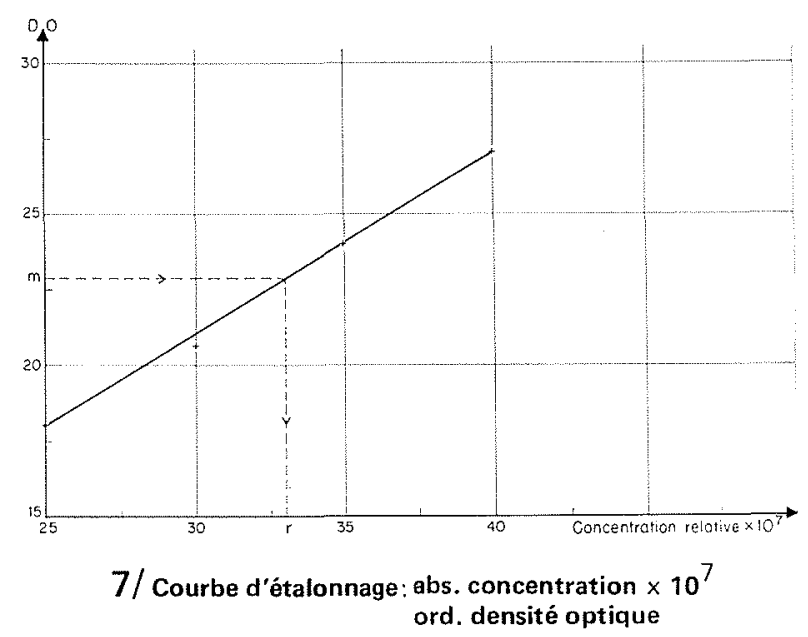

N.B.

1) Si la longueur d'onde optimale est inconnue, on peut la déterminer facilement et rapidement. Préparer une solution diluée du colorant (quelques milligramme au litre) et lire la déviation obtenue aux diverses longueurs d'onde. Adopter pour l'étude la longueur d'onde qui donne la déviation maximale. Cet essai rapide nous a conduit à utiliser, pour le Jaune Soleil, une longueur d'onde légèrement inférieure à celle indiquée : $470 \mathrm{~nm}$ au lieu de $483 \mathrm{~nm}$.

2) Si le débit approché de la rivière n'est pas connu, préparer par dilutions successives des solutions dont les concentrations sont dans un rapport simple (puissance de 10 ou multiples de ces puissances) avec celle de la solution injectée. A partir des deux solutions qui encadrent les échantillons recueillis au point de prélèvement, resserrer l'intervalle des rapports de concentration en diluant convenablement la solution la plus concentrée.

3) Les matières en suspension faussent le résultat des lectures, il est indispensable de les éliminer, ce qui peut être fait sans inconvénient par filtration sur verre fritté sous vide de la trompe à eau.

\section{Critique de la méthode aux colorants alimentaires}

Il serait souhaitable que la nouvelle méthode proposée incitât les spécialistes du jaugeage chimique à formuler des remarques, dont la prise en compte ne pourrait que permettre des améliorations. Dès à présent, on peut noter un certain nombre d'avantages et d'inconvénients par rapport à la méthode au dichromate de sodium.

\subsection{Avantages de la nouvelle méthode}

a) Elle ne met en ouvre aucun produit toxique.

b) L'emploi de la fluorescéine comme traceur préliminaire peut être évité.

c) Les opérations de laboratoire sont simplifiées.

d) La précision est au moins aussi bonne que celle des méthodes classiques. 
e) Les colorants alimentaires préconisés sont parfaitement stables. Aucun signe de dégradation n'a pu être observé lors de séjours de plusieurs heures en eau de rivière propre ou moyennement chargée.

f) Bien que nous n'ayons effectué aucun essai dans ce domaine, on peut certainement utiliser les colorants alimentaires en jaugeage chimique par intégration, ce qui est d'une importance considérable étant donné le danger que présente pour la faune piscicole l'injection brutale de grandes quantités de dichromate de sodium dont la toxicité se fait sentir pendant un temps et sur une distance non négligeables.

\subsection{Inconvénients de la nouvelle méthode}

a) Elle nécessite, plus qu'avec le dichromate de sodium, un spectrophotomètre sensible. L'équipement progressif des laboratoires de "Qualité des Eaux" doit conduire peu à peu à satisfaire cette exigence.

b) Les colorants alimentaires sont moins solubles que le dichromate de sodium : généralement de 80 à $100 \mathrm{~g} .1^{-1}$ contre environ $600 \mathrm{~g} \cdot 1^{-1}$, ce qui conduit à injecter des quantités de solution nettement plus grandes. Toutefois, la disposition d'un spectrophotomètre de bonne qualité permet de supporter une plus grande dilution finale qu'avec le dichromate de sodium, ce qui compense partiellement cet inconvénient. D'autre part, on peut, comme il a été dit, porter la concentration mère à $200-250 \mathrm{~g} / 1$ en utilisant au lieu d'une solution une suspension fluide qui se transforme en solution dès son arrivée dans le cours d'eau.

c) Les colorants alimentaires se dissolvent moins facilement que le dichromate de sodium, dont la dissolution est immédiate, au moins aux concentrations faibles ou moyennes. L'adjonction d'un surfactif doit permettre de parer à cet inconvénient. Des essais sont en cours.

d) Le prix des colorants alimentaires est plus élevé que celui du dichromate de sodium.

Dès à présent, l'utilisation de colorants alimentaires constitue un progrès considérable sur le plan de la qualité des eaux, et non négligeable sur celui de la simplification du procédé d'exploitation en laboratoire.

En l'état actuel de sa mise en ouvre, la méthode est déjà parfaitement adaptée aux jaugeages des débits faibles et moyens, disons jusqu'à une dizaine de mètres cubes par seconde. Sa parfaite innocuité est particulièrement intéressante pour la mesure des débits d'étiage.

e) Etant donné les nouvelles plus ou moins fantaisistes qui ont été diffusées au cours des derniers mois à propos des autorisations d'utilisation des colorants alimentaires, il n'est peut-être pas inutile de préciser ce qu'il en est.

Un arrêté du 24 août 1976 du Ministère de l'Agriculture, paru au J.O. du 28 août, interdit à compter du ler octobre 1977 la vente de denrées alimentaires contenant un ou plusieurs des neuf colorants suivants :

$\begin{array}{ll}\text { E } 103 \text { chrysoïne } S & \text { jaunes } \\ \text { E } 105 \text { jaune solide } & \\ \text { E } 111 \text { orangé GGN } & \text { orange } \\ \text { E } 121 \text { orseille, orcéine } & \\ \text { E } 125 \text { écarlate G N } & \text { rouges } \\ \text { E } 126 \text { ponceau 6 R } & \end{array}$

\section{E 130 bleu solanthrène $\mathrm{R} S$ bleu \\ E 152 noir 7984 noir \\ E 181 terre d'ombre brûlée}

Le même arrêté limite les conditions d'emploi d'un autre colorant

\section{E 123 amarante}

rouge

Aucune mesure ne vise les trois colorants que nous proposons, et aucun projet de réglementation ne les concerne. Par ailleurs, si un colorant utilisé en jaugeage venait à être interdit, il est bien évident que son usage en jaugeage resterait d'une innocuité totale, en particulier vis à vis d'autres traceurs nettement toxiques comme le dichromate. Si sa fabrication en tant que colorant alimentaire venait à être interdite, il resterait toujours la possibilité d'utiliser le produit brut dont il est originaire et qui, quoique n'ayant pas la qualité de colorant alimentaire, serait aussi inoffensif pour une utilisation en jaugeage.

Si cette utilisation des colorants alimentaires intéresse les services hydrométriques et que l'on passe du stade des essais au stade pré opérationnel, il faut souhaiter que ces services obtiennent à terme, des producteurs, une qualité brute ("technique") c'est-à-dire non purifiée de ces colorants qui n'auraient alors plus droit au qualificatif "alimentaire" mais qui pourraient être bien meilleur marché.

\section{Remerciements}

Pour terminer, nous tenons à remercier tous ceux qui nous ont permis de réaliser cette étude.

En premier lieu, nous exprimons tout particulièrement notre gratitude à $\mathrm{M}$. Hlavek, Ingénieur en Chef du Génie Rural, des Eaux et des Forêts, Chef de la Division Hydrologie-Hydraulique Fluviale du C.T.G.R.E.F., qui a été l'instigateur de notre travail et a continué à s'y intéresser après son départ de la Division, ainsi qu'à M. Oberlin, Ingénieur Hydrologue, Chef de la Subdivision Hydrologie, qui nous a aidés de sa compétence et par la mise à notre disposition de moyens matériels.

Les études d'orientation effectuées sur les bassins versants représentatifs de l'Orgeval, ont pu ètre menées à bien grâce à l'aide efficace de MM. J.B. Rosique, Chef de la Station de Boissy-le-Chatel, J.L. Rosique et J. RibotBruno, qui ont participé à nos essais, tant sur le terrain qu'au laboratoire.

Nos remerciements s'adressent ensuite à M. Varet, Chef du Laboratoire de Chimie de la Station d'Hydrobiologie d'Aix les Bains du C.T.G.R.E.F., qui a bien voulu, malgré ses charges, mettre à notre disposition son laboratoire et son excellent personnel, en particulier M. Bogey, pour les opérations sur le terrain, et M. Roulier, pour l'exploitation en laboratoire.

Enfin, la dernière série d'essais a pu être effectuée grâce à l'efficace collaboration du Service Régional d'Aménagement des Eaux Languedoc-Roussillon, où nous avons rencontré chez MM. Tartes, Ingénieur Divisionnaire des Travaux Ruraux, Adjoint de l'Ingénieur en Chef du Génie Rural, des Eaux et des Forêts, Directeur du S.R.A.E., et Delmas, Ingénieur Hydrogéologue au S.R.A.E., un esprit 
particulièrement coopératif, qui s'est traduit par leur participation aux opérations, diverses remarques judicieuses et la mise à notre disposition, pendant deux jours, de deux collaborateurs rompus aux méthodes de jaugeage chimique, MM. Guy et Tailhan, dont nous n'avons eu qu'à nous louer.

\section{Bibliographie}

ANDRE (H.) - Méthodes par traceurs utilisés en 1969 pour la mesure des débits liquides. La Houille Blanche (1969), $\mathrm{n}^{\circ} 5$, 535-9.

ARRETE du 10 Août 1961 (J.O. du 26 Août 1961).

BAILLEUX (J.) - Hydrométrie - Jaugeage Chimique - Résultats obtenus sur un dispositif expérimental de tarage de diaphragmes d'injection du traceur. Etude du C.T.G.R.E.F. (1974), $\mathrm{n}^{\circ} 1,34 \mathrm{p}$.

BOUVARD (M.) - Matériel de jaugeage léger (méthode chimique) La Houille Blanche (1950), 466-9.

BURELLI (F.P.), ECREMENT (F.) - La détermination du chrome dans les eaux par spectrophotométrie d'absorption atomique. Chimie Analytique (1969), 51, 11, 561-6 (7 réf.).

CORMARY (Y.) - Emploi d'un traceur coloté dans la mesure des temps de parcours en rivière et applications diverses. Etude bibliographique. Quelques résultats d'expériences. $E d f-D i$ rection des Etudes et Recherches (1968), 37 p.

DODERO (M.) - Modalités pour le titrage des solutions employées dans la méthode chimique pour la mesure des débits. $L a$ Houille Blanche (1953), 8, n $6,883-8$.
Dumas (H.) - La Méthode Chimique pour la mesure du débit des cours d'eau. La Houille Blanche (1952), 690-701.

$$
\text { " (1953), 51-7. }
$$

HLAVEK (R.) - Les bassins versants representatifs de l'Orgeval Généralités. Bulletin Technique du Génie Rural (1967), п1 89 , 42 p.

Directives verbales (1973)

Lettre du 28.12.72 à l'AFNOR - Groupe de Travail ISO/TC 113.

HLAVEK (R.), CAMBON (J.P.), ZUMSTEIN (J.F.) - Les bassins versants représentatifs de l'Orgeval - Historique des Observations. Bulletin Technique du Génie Rural (1968), $\mathrm{n}^{\circ} 90$, $42 \mathrm{p}$

LALLEMAND (A.) - Etude comparée de la fixation de la fluorescéine et de la rhodamine $B$ par trois types de matériaux: craie, sable, marne. B.R.G.M. (1964), DS. 64 - A. 26.

MARICHAL (M.), BENOIT (R.) - Dosage de la rhodamine B dans les eaux naturelles. Chimie Analytique (1962), 44, $70-2$ (2 ref.).

MEINCK (F.) et coll. - Lez eaux résiduaires. Masson(1970), 865 p.

Norme NFT 90-022 Additif - Essais des Eaux - Dosage spectrophotométrique du cuivre aux faibles teneurs.

Norme NFT 90-024 - Essais des Eaux - Dosage spectrophotométrique du manganèse.

PASCAL (P.) - Traité de Chimie Minórale - Tomes I à XX. Masson (1956-1964).

PRUVOT (E.) - Modalités pour le titrage des solutions employées dans la méthode chimique pour la mesure des débits. La Houille Blanche (1953), $389-94$.

RODIER (J.), MARICHAL (M.) -. Contribution à l'étude de la dilution des effluents radioactifs dans le Rhône par le rejet expérimental de rhodamine B. La Houlle Blanche (1961), 636-41.

WATER QUALity CRiteria DATA Book. - U.S. (1971), Vol. 2, $238-42$. 